\title{
MONITORAMENTO DA EXPANSÃO DE POLIURETANAS POR TÉCNICAS DE ANÁLISE DE IMAGEM
}

\author{
E. T. S. ALVES ${ }^{1}$, G. B. RODRIGUEZ ${ }^{2}$, M. K. LENZI ${ }^{2}$, P. H. H. ARAÚJO ${ }^{1}$, M. C. B. COSTA ${ }^{3}$, \\ R. A. IHABUINSKI ${ }^{2}$, L. F. L. LUZ Jr. ${ }^{2}$ \\ ${ }^{1}$ Universidade Federal de Santa Catarina, Departamento de Engenharia Química e Engenharia \\ de Alimentos \\ ${ }^{2}$ Universidade Federal do Paraná, Departamento de Engenharia Química \\ ${ }^{3}$ Universidade Federal do Rio Grande do Norte, Departamento de Engenharia de Materiais \\ e-mail para contato: lenzi@ufpr.br
}

\begin{abstract}
RESUMO - Poliuretanas (PU) apresentam diversas aplicações comerciais, tais como o uso sob a forma de revestimentos e isolantes. Em geral, são formadas pela reação de polióis e isocianatos. A expansão do meio reacional durante o processo de síntese representa um importante aspecto operacional para o projeto da resina, consequentemente, de suas propriedades finais de uso. Desta forma, este trabalho apresenta o uso de técnicas de análise de imagem para o monitoramento do processo de expansão. Os experimentos foram conduzidos considerando a relação mássica de poliol:isocianato em 1:2 e 1:1, sendo realizados em duplicata. Considerando o uso do sistema RGB (Red-Green-Blue) e uma câmera filmadora para análise em tempo real, foi possível monitorar com sucesso a expansão durante o processo de síntese, bem como sua correlação com a perda de massa e aumento de temperatura.
\end{abstract}

\section{INTRODUÇÃO}

As diferentes características químicas e físicas dos poliuretanos são obtidas pela adição de outros compostos químicos que atuam como agentes de expansão, catalisadores, agentes de ligação cruzada, agentes de extensão de cadeia, surfactantes, aditivos, entre outros (Odian, 1991). A escolha dos reagentes para a síntese dos poliuretanos, bem como, a proporção entre eles é responsável pelas diferentes propriedades físicas e químicas do produto final, que irão direcionar as aplicações do mesmo dentro do setor produtivo (Woods, 1987).

Dentre as várias aplicações dos poliuretanos, destacam-se o uso como elastômeros, tintas ou adesivos e espumas rígidas/flexíveis, sendo estas últimas responsáveis pela maior parte do mercado de PU. As espumas rígidas e flexíveis podem ser preparadas por dois processos: i) Processo "one-shot" no qual todos os reagentes são misturados em uma única etapa de mistura (Alves, 2011); ii) O pré-polímero é preparado pela reação de isocianato orgânico com poliois apresentando um teor pré-determinado (estequiométrico) de -NCO livre (Rivera-Armenta et al., 2004). A modelagem matemática das reações de polimerização envolvidas na síntese de poliuretanas é realizada considerando reações de polimerização entre grupos funcionais 


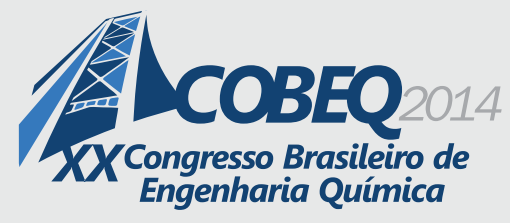

19 a 22 de outubro de 2014

Florianópolis/SC

específicos, como apresentado por Jacobsen e Ray (1992). Estes autores reportam um modelo matemático para a predição do comportamento cinético e da evolução da curva de distribuição de massa molecular, ou seja, modelos em micro, meso e macroescala. Um dos poucos trabalhos reportados na literatura envolvendo o monitoramento da expansão de poliuretanas é apresentado por Khakhar e Harikrishnan (2010) que apresentaram um modelo para formação de espumas flexíveis a fim da predição da abertura de suas células. Os autores utilizaram um sistema adiabático para formação da espuma. Para obtenção dos parâmetros cinéticos da reação foi registrada a temperatura durante o processo de formação. Também foi avaliado pelos autores a variação de densidade da espuma, baseada na perda de peso da espuma durante sua formação. De posse dos resultados cinéticos, densidade e viscosidade foi avaliada a espessura de abertura das células para espumas flexíveis por meio da gravação de vídeo por microscopia eletrônica.

O monitoramento de reações de polimerização apresenta algumas lacunas (Reed e Alb, 2014). O uso de análise de imagens para monitoramento de reações de polimerização foi reportado no controle de tamanhos de partícula em reatores de polimerização em suspensão, por Santos et al (1998), sendo que análises microscópicas do tamanho das partículas foram utilizadas com sucesso para a calibração de equipamento de infravermelho próximo para monitoramento em tempo real por meio de variações de absorbância em determinados picos do espectro. No entanto, a literatura ainda carece de trabalhos que envolvam de análise de imagem para o monitoramento em tempo real da expansão de poliuretanas. Neste sentido, este trabalho apresenta uma proposta de monitoramento usando o sistema de cores RGB (Red-Green-Blue).

\section{MATERIAIS E MÉTODOS}

O aparato experimental usado consistiu de: i) tubo de polietileno transparente com altura de $14 \mathrm{~cm}$ e diâmetro médio de $6 \mathrm{~cm}$, usado como recipiente para formação da espuma; ii) Isocianto MDI e Poliol poliéter (pureza superior a 90\%, Arofibra Resina e Silicones, Curitiba, Brasil); iii) termopares de ferro-constantan, junta exposta de $1 / 8$ ', os quais foram inseridos transversalmente no tubo, sendo um localizado no centro do tubo $(3,0 \mathrm{~cm}$ da parede do tubo) e o outro a uma distância de $1,5 \mathrm{~cm}$ da parede do tubo, porém ambos na mesma altura, como apresentado na Figura 1; iv) Balança analítica de precisão da marca Bioprecisa (FA-2104N); v) câmera de vídeo do tipo webcam Clone ${ }^{\circledR}$ (10028) de 1,3 megapixels de resolução para capturar a imagem da superfície da espuma; vi) placa de aquisição de dados pela Measurements Computing, modelo PMD-1208LS, com canais analógicos de entrada de resolução 12 bits para aquisição do sinal do termopar; vii) Um computador para aquisição e análise dos dados.

Foram realizados dois tipos de experimentos, cujas formulações são apresentadas na Tabela 1. No primeiro tipo, o objetivo foi o monitoramento simultâneo da expansão (altura) e da temperatura e no segundo tipo, o objetivo foi o monitoramento simultâneo da expansão e da densidade da poliuretana. Ressalta-se que o instante inicial da reação foi imediatamente após a mistura dos reagentes, os quais foram alimentados diretamente ao recipiente da polimerização. Observa-se que o ensaio 2 (altura/temperatura) e (altura/densidade) possuem basicamente a mesma formulação, sendo consideradas réplicas para avaliação dos erros experimentais. 


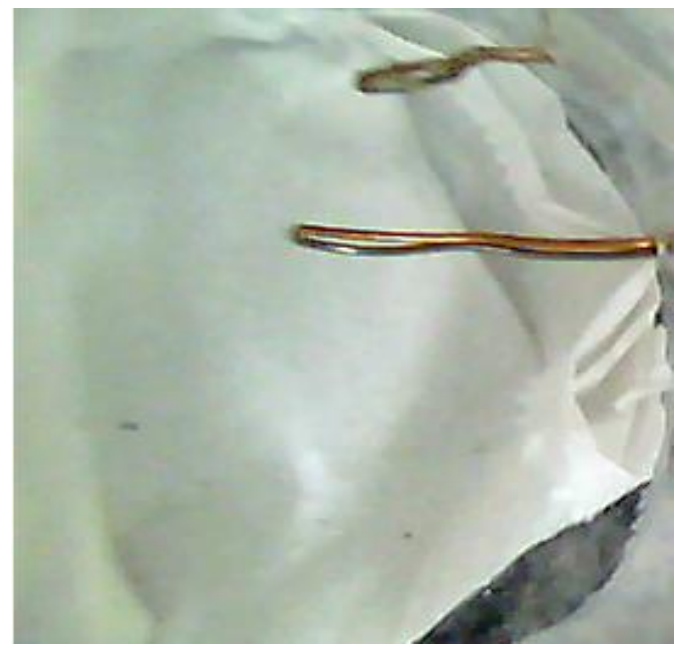

(a)

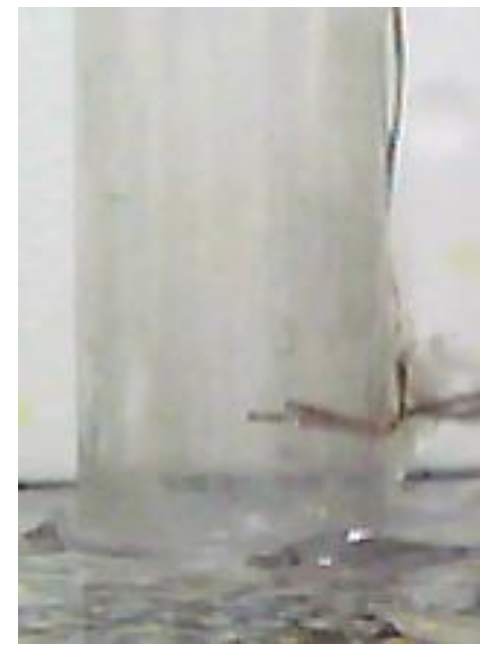

(b)

Figura 1 - Posicionamento dos dois termopares no tubo (a) Vista superior mostrando o posicionamento do termopar em relação ao centro do tubo; (b)Vista lateral mostrando o posicionamento do termopar em relação à base da proveta.

O software utilizado para aquisição e tratamento de imagens foi elaborado conforme o algoritmo apresentado na Figura 2, o qual leva em conta, também, a aquisição em tempo real dos valores de temperatura, por meio do cartão de aquisição de dados.

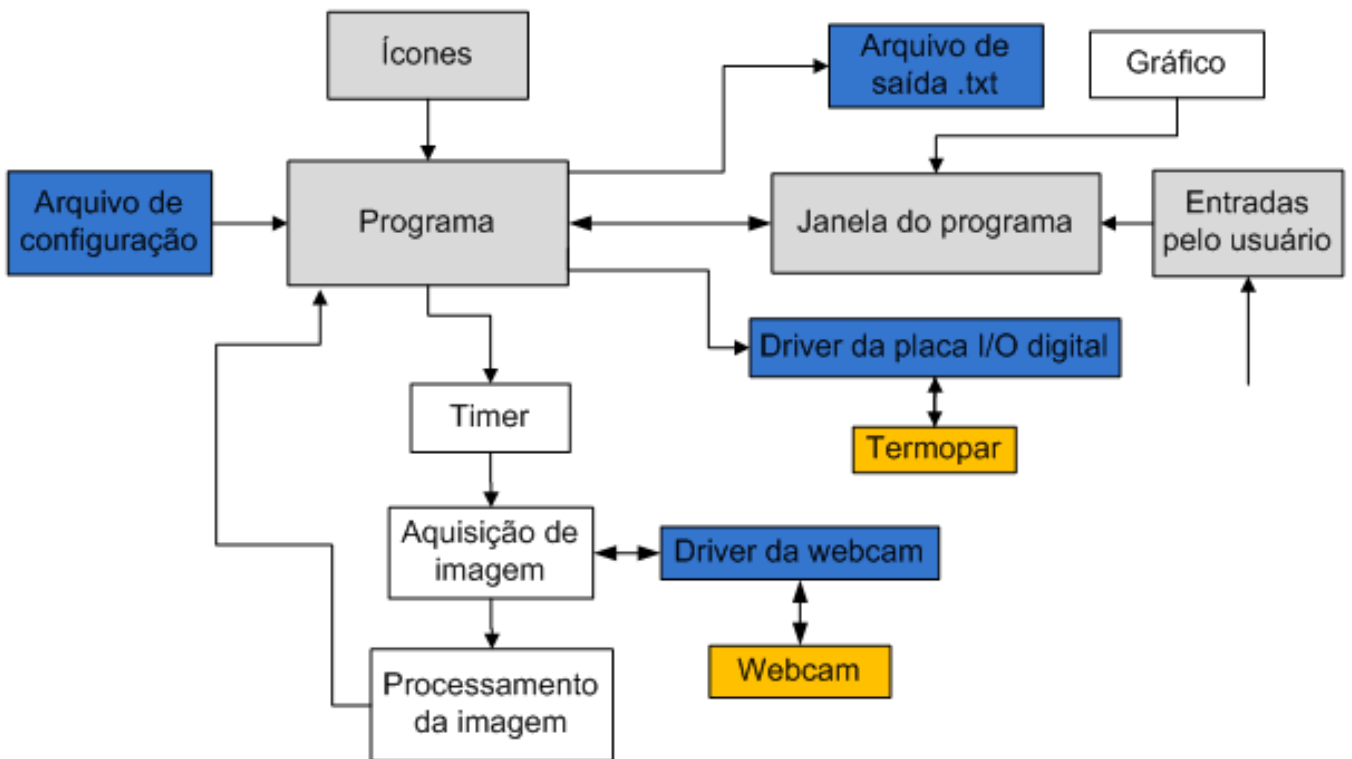

Figura 2- Fluxograma da estrutura do programa desenvolvido para aquisição de imagem para monitoramento da expansão da espuma de PU e registro da temperatura. 
Tabela 1 - Formulação usada para a síntese da Poliuretana

\begin{tabular}{c|cc|cc}
\hline \multirow{2}{*}{ Reagentes } & \multicolumn{4}{|c}{ Massa [g] } \\
\multirow{2}{*}{ Ensaio } & \multicolumn{2}{|c}{ altura/temperatura } & \multicolumn{2}{c}{ altura/densidade } \\
& 1 & 2 & 1 & 2 \\
\hline Poliol & 7,002 & 12,503 & 11,921 & 12,584 \\
Isocianato & 14,128 & 25,235 & 24,147 & 24,046 \\
\hline
\end{tabular}

Foram realizadas análises de IR (infravermelho) do isocianato e do poliol utilizados para fins de caracterização da matéria-prima Foi utilizado um equipamento Thermo Electron Corporation (Modelo Nicolet IR-100 FT-IR). A Figura 3 apresenta o espectro referente ao poliol utilizado, sendo observada a presença do pico característico em $1100 \mathrm{~cm}^{-1}$. A Figura 4, por sua vez, apresenta o espectro referente ao isocianato utilizado, sendo observada a presença do pico característico em $2350 \mathrm{~cm}^{-1}$.

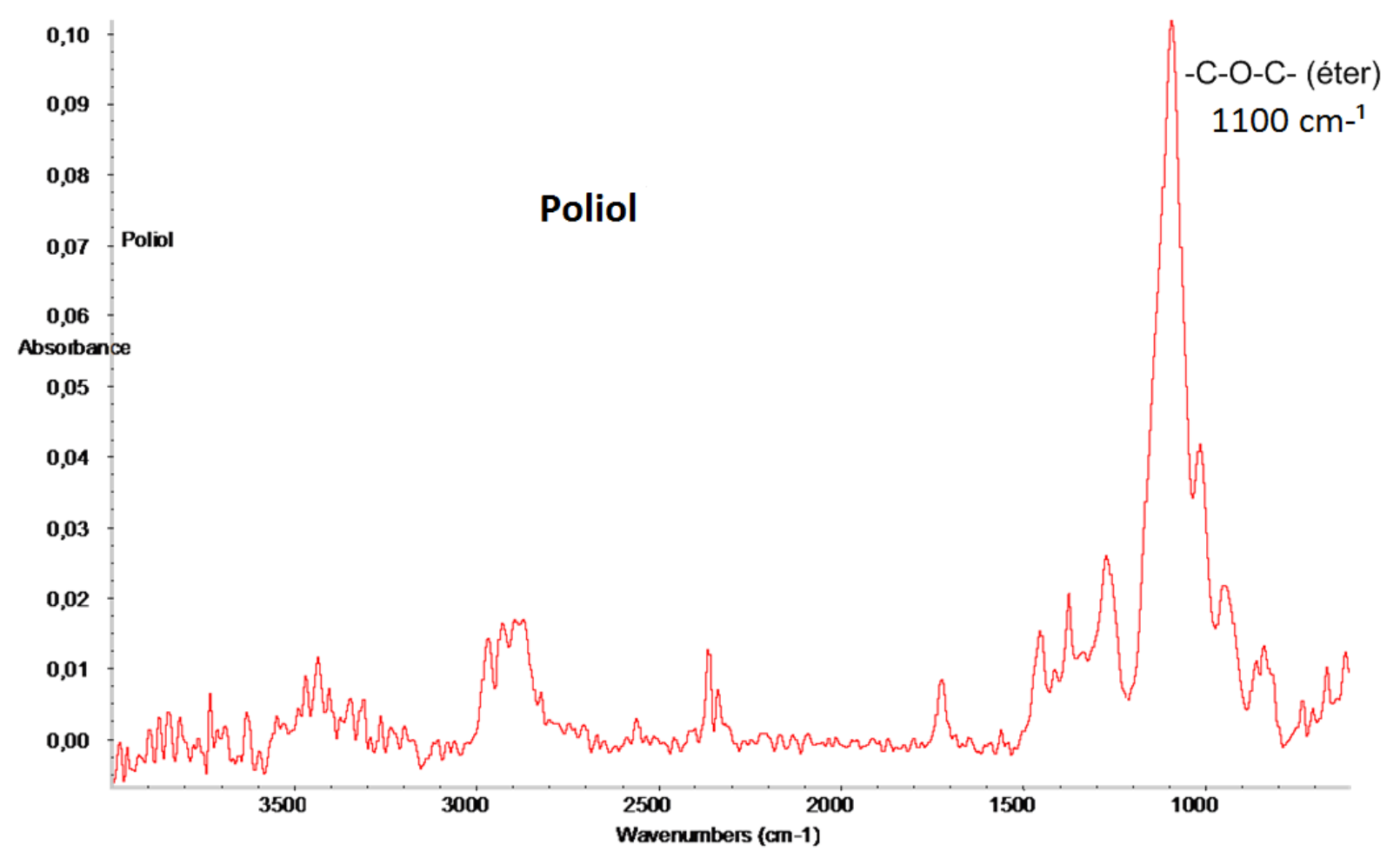

Figura 3 - Espectro de infravermelho do poliol 


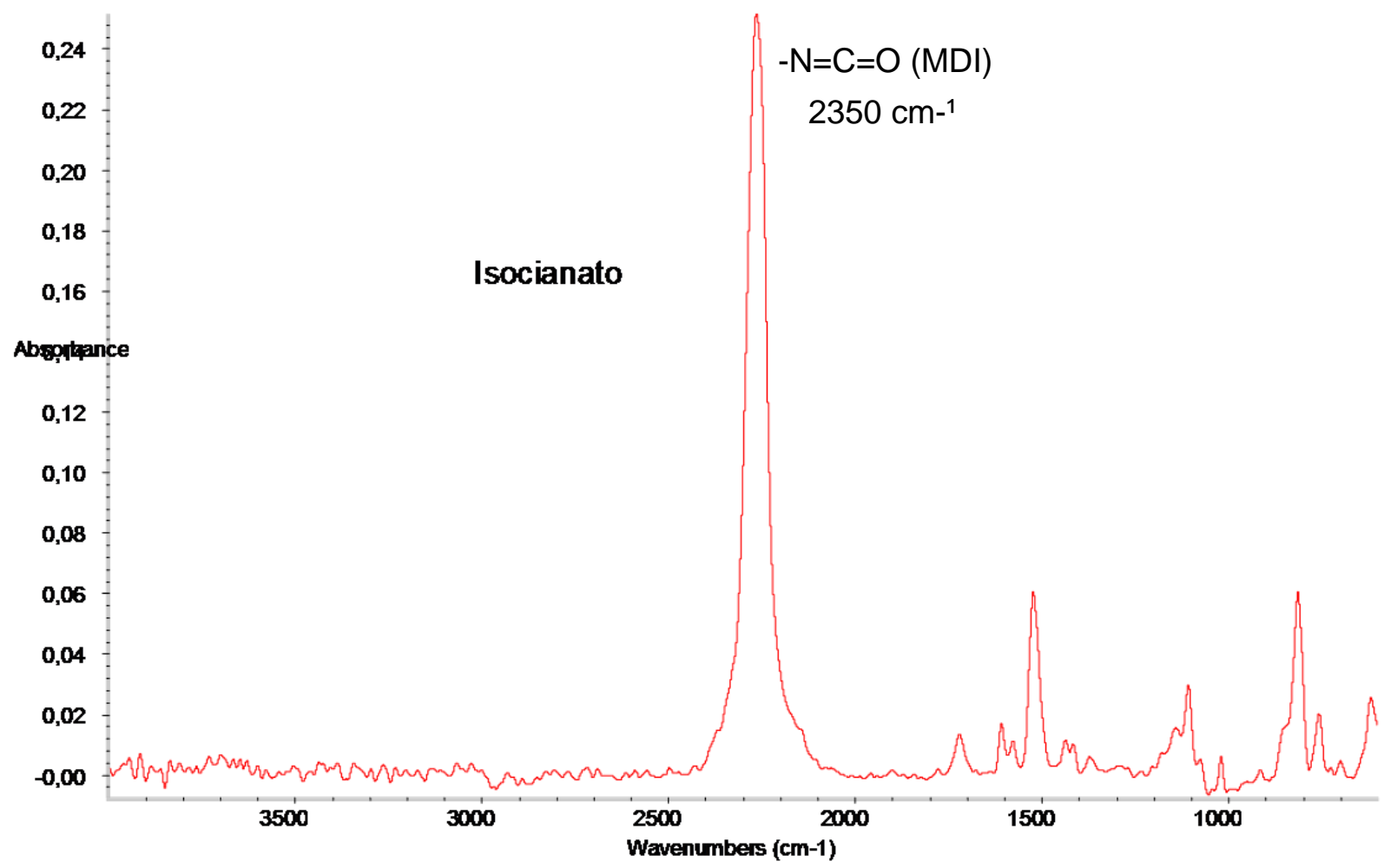

Figura 4 - Espectro de infravermelho do isocianato

\section{RESULTADOS E DISCUSSÕES}

A expansão da espuma e seu aumento da temperatura durante a sua formação, medidos a partir do tempo de creme são mostrados na Figura 5, referente ao ensaio 1 e ao ensaio 2 (altura/temperatura). Nota-se que o crescimento da espuma é adequadamente monitorado, assim como a evolução da temperatura, mostrando que a polimerização é exotérmica. Observa-se que no ensaio 1 a reação de formação da espuma é mais rápida comparada a reação do ensaio 2 , fato relacionado a quantidade de reagentes utilizados. O ensaio 2 ocorreu com o dobro de massa dos reagentes, porém foi mantida a proporção 2:1. O comportamento dinâmico da temperatura é descrito pela apresentação dos valores medidos no gráfico da Figura 5, referindo-se à temperatura do centro do tubo, pois o segundo ponto de aquisição de temperatura não apresentou variações significativas em relação ao ponto do centro do tubo. A Figura 6 apresenta uma imagem obtida do software de monitoramento da expansão, sendo observados os limites superior e inferior, bem como uma linha intermediária, a qual indica a altura instantânea, medida em tempo real. 


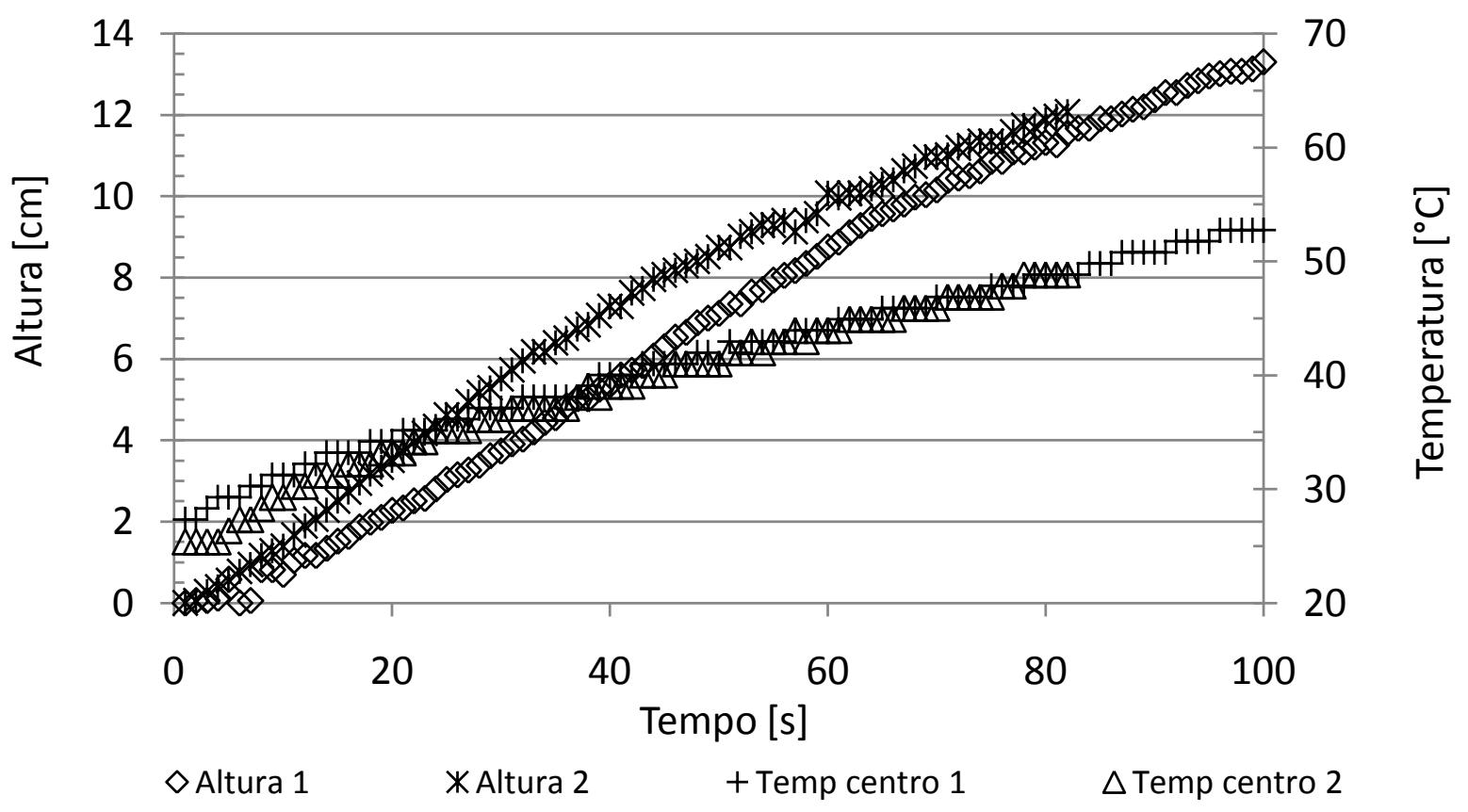

Figura 5 - Comportamento dinâmico da altura e temperatura durante a polimerização.

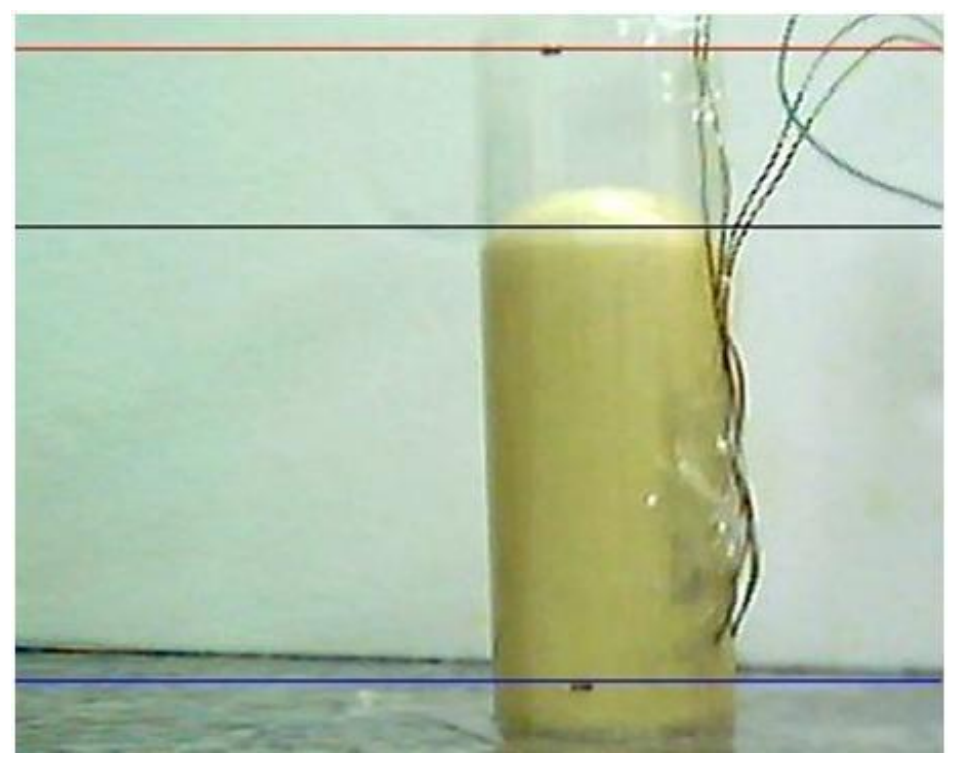

Figura 6 - Imagem obtida durante a polimerização que foi usada para o monitoramento da expansão (altura). 
A Figura 7 mostra a variação na altura e a mudança na densidade da espuma em função do tempo de polimerização. Estas propriedades foram medidas a partir do tempo de creme (instante considerado como início da polimerização) e estão baseadas nos dados de perda de peso e da técnica de análise de imagem. A diminuição na densidade está relacionada a perda de massa medida durante a ascensão da espuma, devido ao aumento na força de empuxo aplicada pelo crescente volume de ar deslocado. Finalmente, pode-se verificar a boa reprodutibilidade dos resultados experimentais.

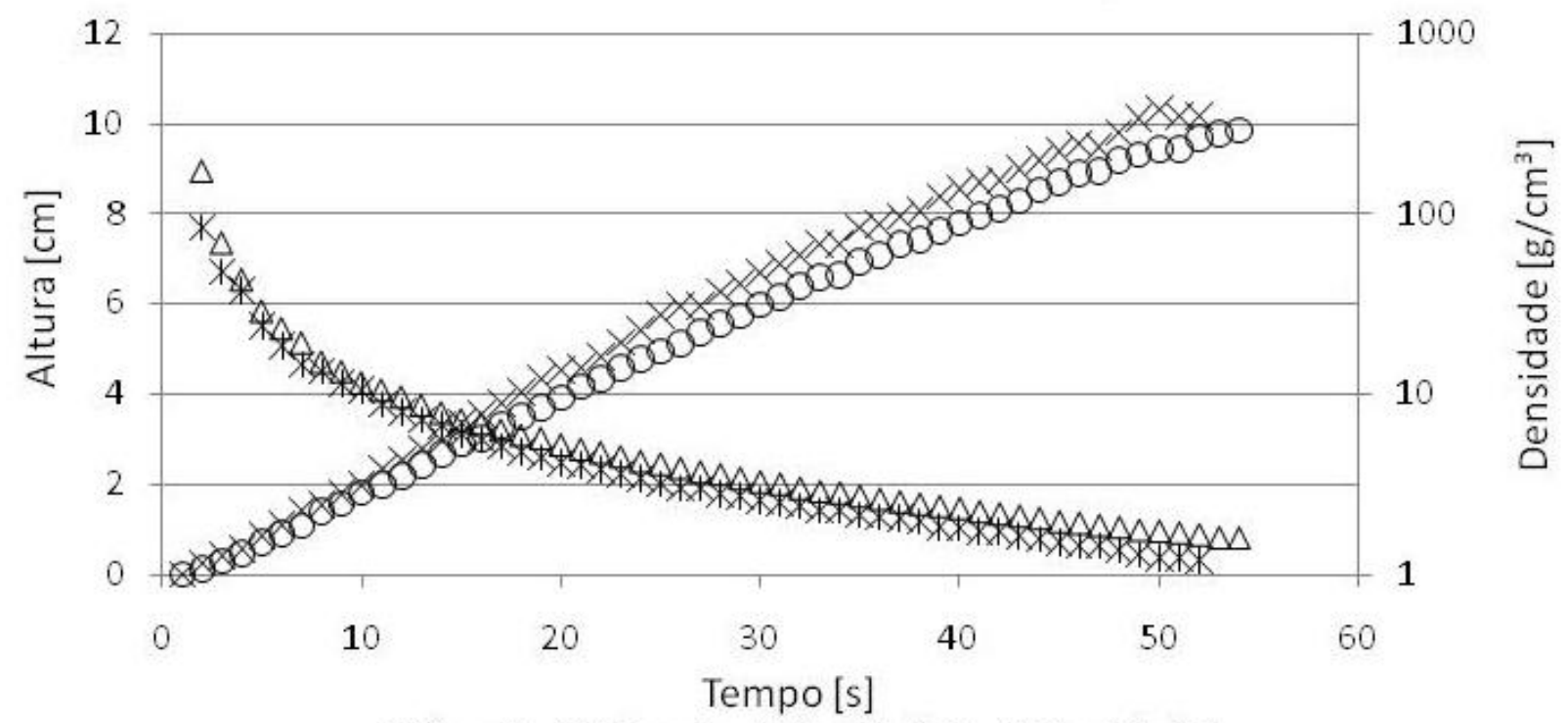

OAltura $1 \times$ Altura $2 \Delta$ densidade $1 \%$ Densidade 2

Figura 7 - Comportamento dinâmico da altura e densidade durante polimerização.

\section{CONCLUSÕES}

Técnicas de análise de imagem podem ser usadas para o monitoramento da expansão de poliuretanas em tempo real durante seu processo de síntese (polimerização). O sistema de cores RGB pode ser utilizado com sucesso para quantificar a expansão devido à diferença de cores ao longo do sistema reacional durante a polimerização. Destaca-se que esta expansão pode ser adequadamente correlacionada à perda de massa do sistema, bem como ao aumento da temperatura.

\section{AGRADECIMENTOS}

Os autores agradecem à CAPES e CNPQ pelo apoio financeiro e bolsas de estudo. 


\section{REFERÊNCIAS}

ODIAN, G. O. Principles of polymerization. New York: John Wiley \& Sons, 1991.

WOODS, G. The ICI poliurethanes book. New York: John Wiley \& Sons, 1987.

ALVES, E. T. S. Alicação de técnicas de análise de imagem para sistemas de engenharia química. Dissertação de mestrado, Universidade Federal do Paraná, 2011.

RIVERA-ARMENTA, J. L.; HEINZE, T.; MENDOZA-MARTINEZ, A. M. New polyurethane foams modified with cellulose derivatives. Euro. Pol. J., v. 40, p. 2803-2812, 2004.

JACOBSEN, L. L.; RAY. W. H. Unified modeling for polycondensation Kinetics. J. Macromol. Sci. Rev. Macromol. Chem. Phys., v. C32 (3 \& 4), p. 407-519, 1992.

KHAKHAR, D. V.; HARIKRISHNAN, G. Modeling the dynamics of reactive foaming and film thinning in polyurethane foams. AIChE J., v.56, p. 522-530, 2010.

REED, W. F., ALB, A. M., Monitoring polymerization reactions: from fundamentals to applications, New York: John Wiley \& Sons, 2014.

SANTOS, A. F.; LIMA, E. L.; PINTO, J. C. In-line evaluation of average particle size in styrene suspension polymerizations using near-infrared spectroscopy, J. Appl. Polym. Sci, v. 70, p. 1737-1745, 1998. 